\title{
Optimal management of venous thromboembolism in adolescent and young adult oncology patients
}

This article was published in the following Dove Press journal:

Clinical Oncology in Adolescents and Young Adults

6 September 2016

Number of times this article has been viewed

\section{Sarah McKillop' \\ Cynthia Wu² \\ Aisha Bruce' \\ Joseph Brandwein ${ }^{2}$}

'Division of Immunology, Hematology, Oncology, Palliative Care and

Environmental Medicine, Department of Pediatrics, ${ }^{2}$ Division of Hematology, Department of Medicine, University

of Alberta, Edmonton, AB, Canada
Correspondence: Joseph Brandwein Division of Hematology,

Department of Medicine, University of Alberta,

II 350, 83 Avenue,

Edmonton, AB T6G 2G3, Canada

$\mathrm{Tel}+\mathrm{I} 7804077482$

Fax +I 7804072680

Email jbrandwe@ualberta.ca
Abstract: Venous thromboembolism (VTE) is a serious complication experienced by adolescents and young adults (AYAs) diagnosed with cancer. Data exist in the adult literature to guide the management of cancer-associated thrombosis. Unfortunately, little is known regarding the epidemiology of cancer-associated thrombosis in AYAs. As a result, evidence on the treatment and prevention of thrombosis in this vulnerable population is lacking, posing a great challenge to physicians caring for AYAs with cancer. It is clear that the basic principles of VTE likely apply to AYAs and that low-molecular-weight heparin is the drug of choice for the treatment of VTE regardless of age. We review the available data on the epidemiology, diagnostic methods, and management of AYAs with cancer and VTE. Recognizing the lack of accepted guidelines for the prevention or management of VTE in this population, we offer expert opinion recommendations to serve as guidance to improve management of thrombosis in AYA cancer patients. Keywords: adolescents, young adults, thrombosis, cancer, venous thromboembolism, anticoagulation

\section{Introduction}

For almost 200 years, an association between thrombosis and cancer has been recognized. ${ }^{1,2}$ Of thrombotic complications encountered by patients with cancer, venous thromboembolism (VTE) is the most common. ${ }^{3}$ Most of our knowledge on cancerassociated thrombosis is derived from adult data as VTE is the second leading cause of death in this population ${ }^{4}$ and is associated with significant morbidity. VTE risk, associated comorbidities, and presenting cancer types vary significantly by age, and management plans applicable to the adult population may not be relevant to younger patients.

The adolescent young adult (AYA) oncology population is defined as individuals diagnosed with cancer between the ages of 15 and 39 years. ${ }^{5}$ Unfortunately, current international literature and treating institutions have various definitions of the age range for an AYA oncology patient, making it difficult to clearly define epidemiological and treatment data. Additionally, AYAs with cancer are not treated universally in the same environment. The approach to the treatment of the malignancy and thrombosis is often driven by institutional protocols and usually follows either pediatric or adult protocols depending on the center's age cutoff for "adulthood". For AYAs receiving treatment in an adult program, it is more likely they will be managed according to adult guidelines despite the risk and benefits of anticoagulation to AYAs not being clearly understood. 
The epidemiology and etiology of VTE among AYAs with cancer, as well as the best management practices for both prophylaxis and treatment of a deep vein thrombosis (DVT), have not been well elucidated. This review summarizes the existing body of knowledge regarding VTE in AYA oncology patients and attempts to place this into rational principles that may help to guide management.

\section{Epidemiology}

The annual incidence of VTE increases with age, ranging from less than $0.5 / 1,000$ in those under 15 years of age to approximately $1 / 1,000$ in the general adult population, increasing to $1 / 100$ in the geriatric population..$^{6-10} \mathrm{~A}$ disturbing trend of significantly increasing rates of VTE in the pediatric and adolescent population has been recently documented. Among pediatric patients, adolescents were reported to have the highest incidence of VTE at 94 cases per 10,000 hospital admissions, and those with a malignancy had the highest rate of first time and subsequent VTE. ${ }^{11-15} \mathrm{~A}$ similar trend has been reported in the adult literature, with a notable increase in the rates of VTE in adults with cancer over the last several decades. ${ }^{16}$ Approximately $20 \%$ of reported cases of VTE among adults occur in patients with cancer. ${ }^{17,18}$

For AYAs with cancer, only one retrospective review exists for all types of cancer-associated VTE. O'Brien et al ${ }^{19}$ determined that for 15-24-year olds treated for cancer at children's hospitals throughout the US between 2001 and 2008 , VTE occurred in $5.3 \%$ of the 9,721 patients reviewed. The odds ratio of VTE increased with age. Those aged 18-20 years had an odds ratio of 1.65 (95\% confidence interval, $1.36-2)$, and those aged $21-24$ had an odds ratio of 1.67 (95\% confidence interval, 1.21-2.32), when compared to those aged 15-17 years. AYAs with leukemia, lymphoma, and bone and soft tissue tumors had the highest odds ratios of VTE $(5.53,4.83$, and 4.32 , respectively) when those with brain tumors were used as a reference group.

Smaller site-specific or disease-specific studies provide further insights into the epidemiology across the AYA age and cancer diagnosis spectrums. Panessha et $\mathrm{al}^{20}$ reported that the rate of cancer-related VTE managed in outpatient DVT clinics in the UK for AYAs between 18 and 31 was $2.6 \%$. Kayser-Gatchalian and Kayser ${ }^{21}$ reported a higher incidence of VTE for patients aged 20-39 years with intracranial tumors compared to those aged $10-19$ years. Audino et $a^{22}$ demonstrated a statistically significant increased incidence of thrombosis in the AYA population, defined as 15-28 years old, when compared to their younger peers. Similarly, for patients with acute lymphoblastic leukemia (ALL), the
Dana Farber Consortium reported VTE rates of $18 \%-20 \%$ for patients aged 11-20 years using their asparaginase-intensive protocols, increasing to $25 \%$ for those aged $21-30$ years and $42 \%$ for patients over 30 years of age. ${ }^{23}$ This contrasted with rates of $2 \%-7 \%$ for ALL patients aged 10 years and below.

For AYAs with sarcoma, limited data regarding the risk of thrombosis are available. Paz-Priel et al ${ }^{24}$ found an overall VTE rate of $16 \%$; the incidence was higher at $23 \%$ for those presenting with metastatic disease, as compared to $10 \%$ for those with localized disease. This finding of increased thrombosis in the presence of metastatic disease has also been identified in adult epidemiology studies for a variety of tumor types. ${ }^{3,25}$

\section{Pathophysiology and risk factors}

The pathophysiology behind the development of VTE in patients with cancer is complex. In adult patients with cancer, changes in the hemostatic system have been demonstrated, ${ }^{26}$ with evidence of chronic hemostatic activation frequently observed even in the absence of overt VTE. ${ }^{27}$ Considered classically, the development of thrombosis among AYAs with cancer can be explained through abnormalities in Virchow's triad including venous stasis, vascular injury, and hypercoagulability. ${ }^{28}$ Venous stasis can occur during periods of immobility such as prolonged hospitalization associated with cancer treatment and/or complications. Occasionally, the location of a tumor may also disrupt normal lymphatic or blood flow. Vascular injury can occur after surgical intervention for tumors, tumor invasion of vascular structures, and via local irritation from central venous catheters used to deliver chemotherapy, blood products, and transfusions. The use of indwelling central venous catheters is associated with a $5 \%-12 \%$ incidence of catheter-related upper extremity thrombosis. ${ }^{29}$

The cancer tumor burden may contribute to a hypercoagulable state through acute phase reactions, but tissue necrosis, changes in hemodynamic states and abnormal metabolism of proteins, and the prothrombotic properties of tumor cells themselves, most likely contribute to the development of hypercoagulability in patients with cancer. Superimposed on these inherent tumor-related factors are treatment-related factors. These include chemotherapy agents that are well recognized to be associated with an excess rate of thrombosis. Asparaginase, a key drug in the treatment of ALL in AYAs, decreases hepatic production of anticoagulants including antithrombin, ${ }^{30}$ leading to a hypercoagulable state and an increased risk of VTE in both pediatric and adult patients. ${ }^{31-33}$

Using these recognized mechanisms of thrombosis, risk factor-based tools have been developed in the adult cancer 
population; these include the National Comprehensive Cancer network ${ }^{5}$ and the Khorana predictive model for chemotherapy-associated VTE risk assessment. ${ }^{34}$ In the pediatric cancer population, the Prentiss Pediatric VTE Risk assessment tool has been validated for use in inpatient units and some have extrapolated its use to AYAs with cancer. ${ }^{35,36}$ On the basis of this tool, AYA oncology patients up to the age of 31 years at high risk for VTE include those with a central venous catheter, a past VTE, having undergone an orthopedic procedure, having a septic episode, and being obese. However age, type of cancer, and the use of specific therapeutic agents such as asparaginase and corticosteroids were not included in the risk score.

\section{Diagnostic evaluation of thrombosis}

There are no VTE diagnostic algorithms specific to AYA populations or cancer populations. For the pediatric population, there is limited research on the appropriate modality for assessment of VTE. Recognizing that the overall prevalence of asymptomatic VTE in children with cancer is reported to be as high as $40 \%,{ }^{37}$ modalities used to diagnosis VTE in pediatrics include ultrasound, venography, computed tomography, and magnetic resonance imagining. ${ }^{38}$ The role of D-dimer as a predictive tool in the diagnosis of VTE has not been validated in pediatric patients. ${ }^{39}$ On the basis of the higher incidence of upper extremity VTE in the pediatric oncology population, generally associated with the use of a central venous catheter, an evidence-based recommendation for the detection of upper venous system thrombosis is available. ${ }^{38}$ The PARKAA study concluded that both a venogram and ultrasound in combination were needed since venography was more sensitive than ultrasound in detecting intrathoracic (central) VTE, but ultrasound was more sensitive for internal jugular and axillary VTE. ${ }^{40}$ There are limited data supporting the use of three-dimensional gadolinium-enhanced magnetic resonance imaging for detecting pediatric upper venous thrombosis; however, there have been no comparative studies with currently recommended ultrasound and venography to establish improved efficacy. ${ }^{41}$ The current recommendations for diagnosing VTE in the lower venous system by ultrasound and the diagnosis of pulmonary embolism (PE) in pediatrics using isotope lung scanning and computed tomography pulmonary angiography lack evidence in pediatrics but are extrapolated from adult literature.

Extensive research has gone into the development and validation of tools that can be used to accurately diagnose lower extremity DVT and PE in adults. Many predictive tools exist, but the most widely recognized is the Wells score, used for both $\mathrm{DVT}^{41}$ and PE. ${ }^{42}$ Incorporation of these tools into a diagnostic algorithm suggests that either D-dimer testing or diagnostic imaging further improves sensitivity and specificity. ${ }^{43}$ For DVT, the accepted modality is now the compression ultrasound (validated against venogram, the prior gold standard $)^{44}$ and for PE, either spiral computed tomography of the chest ${ }^{45}$ or ventilation/perfusion lung $\operatorname{scan}^{46}$ (validated against the pulmonary angiogram, the prior gold standard). This diagnostic algorithm has not been specifically validated in the cancer population.

While adult or pediatric models may not apply to AYA patients, general principles of requiring clinical suspicion for VTE, algorithms to reduce unnecessary testing and maximize necessary testing, and diagnostic imaging studies of high quality still apply to all patients of any age. Patients aged $\geq 18$ years would have been included in the aforementioned diagnostic trials in adults, and thus it would stand to reason that these algorithms can be applied to older AYA populations. Physiologic and anatomic properties of younger AYAs may be varied enough to warrant further validation of these tools in these patients.

\section{Management of VTE}

There are currently no recommendations for the prevention or management of VTE in the AYA oncology population. On the basis of the institution where the AYA is receiving treatment, either pediatric or adult guidelines are generally used regardless of patient age. Risk assessment tools designed to guide prophylaxis and treatment of VTE have been developed for both the adult and pediatric population; however, validated tools for AYAs with a diagnosis of cancer are lacking. On the basis of available data from studies in these populations, a recommended management algorithm is outlined in Figure 1, and a detailed discussion and rationale are provided.

\section{Basic principles of VTE prevention and management}

Certain principles likely can apply to patients of any age regardless of medical diagnosis. With regard to AYA oncology, until studies specifically addressing this population are available, recommendations and principles for practice need to be extrapolated from either the pediatric or adult thrombosis literature. A review of some of these principles with reference to adult literature where there is a significantly greater wealth of data in general VTE treatment as well as cancer-associated thrombosis treatment follows, but two important principles need to be recognized: 


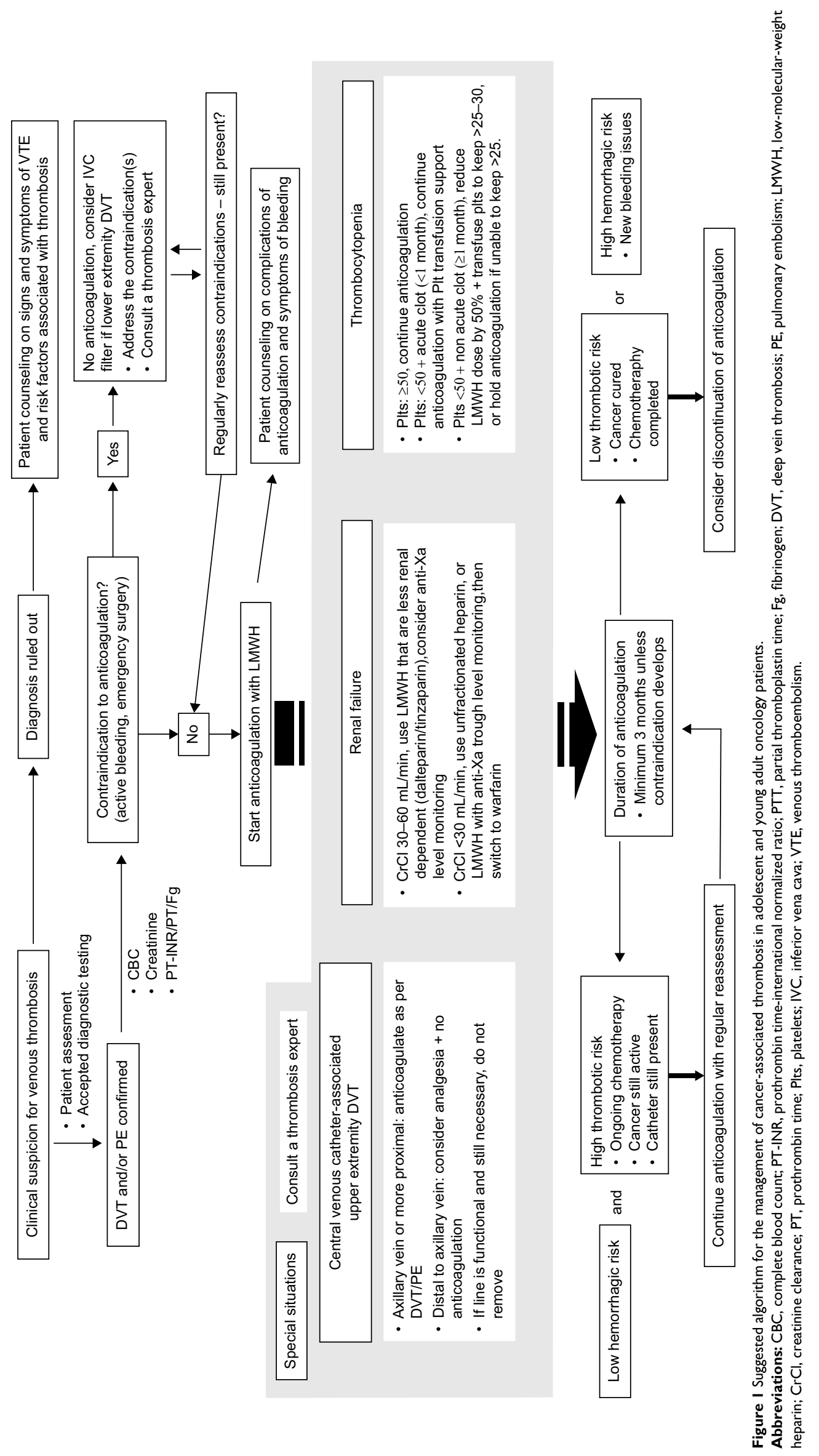


First, while there is a greater proportion of incidentally noted VTE in cancer populations, most VTE are symptomatically diagnosed. ${ }^{47}$ Symptoms of VTE impact patient morbidity and quality of life. Incidental proximal VTE in adult cancer patients carries the same prognosis as symptomatically diagnosed VTE. ${ }^{47}$ More studies in AYA and pediatric populations would be required to confirm this finding in younger patients. Treating acute symptomatic VTE with anticoagulation can reduce the risk of clot progression and embolization. In the adult population, anticoagulation with at least 3 months is associated with a recurrent VTE risk reduction of $>90 \%{ }^{48}$ Second, VTE is associated with mortality. Up to $10 \%$ of adult PE patients die within the first hour of presentation. ${ }^{45}$ VTE is also the second leading cause of death in adult cancer patients. ${ }^{4}$ Similar data are not available for pediatrics or AYAs with cancer. Based on these first two principles, it would stand to reason that an acute DVT or PE deserves anticoagulant therapy in all patients even if the exact risk numbers may vary somewhat between age groups. Consideration of active hemorrhage and other high-risk situations may alter treatment decisions.

\section{What is the role of thromboprophylaxis?}

The routine use of thromboprophylaxis for hospitalized adults has not been validated for the AYA population. There is currently no role for routine thromboprophylaxis in the pediatric oncology population, ${ }^{49,50}$ and the role of routine thromboprophylaxis for ambulatory patients on chemotherapy is still debated for adult patients with cancer. Validated risk assessment tools for thrombosis in AYAs diagnosed with cancer, as well as prospective collaborative studies directed at developing prediction models to identify AYAs at risk for VTE, are needed to better understand the role of routine thromboprophylaxis in this population.

Having said this, appropriate thromboprophylaxis with low-molecular-weight heparin (LMWH) should be considered in higher-risk situations (eg, perioperative, decreased mobility associated with hospitalization) in the absence of contraindications, in accordance with recently published adult guidelines for cancer patients. ${ }^{51}$

\section{What is the anticoagulant of choice for treatment of VTE?}

With regard to the treatment of diagnosed VTE in the AYA patient with cancer, there is consensus from both expert opinion in the pediatric literature and evidence-based studies in the adult literature that LMWH is the preferred anticoagulant. ${ }^{39,52-54}$ Unfortunately, randomized control trials in pediatric anticoagulation are few, leaving much of the pediatric thrombosis literature as expert opinion-based and with extrapolation from the adult literature. Monagle et al ${ }^{52}$ suggest that, in the pediatric oncology patient, oncology therapies are generally intensive and associated with significant periods of thrombocytopenia that increase bleeding risks. Since AYA oncology therapies are frequently modeled from the more intense pediatric protocols due to increased patient treatment tolerances with the goal of improved survival, ${ }^{5,56}$ consideration into balancing the bleeding risk in this population must be made.

In the adult cancer populations, LMWH, a parenteral anticoagulant with no direct drug-drug or food-drug interactions, has been shown in the CLOT trial to be superior to warfarin in patients with VTE and active cancer, with a $50 \%$ VTE recurrence risk reduction using dalteparin versus warfarin. ${ }^{57}$ Various other trials have confirmed this finding, including a subgroup analysis of the LITE trial ${ }^{58}$ and the more recent $\mathrm{CATCH}$ trial. ${ }^{57}$ The $\mathrm{CATCH}$ trial demonstrated an $\sim 30 \%$ VTE recurrence risk reduction with tinzaparin versus warfarin but did not meet statistical significance $(P=0.07)$. It should be noted that patients enrolled on the $\mathrm{CATCH}$ trial were less likely to be on chemotherapy or have metastatic disease and had a better baseline Eastern Cooperative Oncology Group status and lower mortality compared with patients in CLOT trial. This is reflected in the lower rate of recurrent VTE in the warfarin arm of CATCH (10.5\%) compared to CLOT (15.8\%), despite a similar rate of recurrent VTE in their LMWH arms (7\%-8\%). A 30\% risk reduction in over 900 patients is still supportive of LMWH in cancer-associated thrombosis, though results of the $\mathrm{CATCH}$ trial raise the question of whether this difference disappears in cancer patients who are not on active chemotherapy and who are experiencing less morbidity.

In pediatrics, the REVIVE study, a randomized control trial of 78 children, included $30 \%$ pediatric patients with cancer. The use of LMWH compared to unfractionated heparin transitioned to warfarin trended toward less recurrence of clot and fewer bleeding complications in the LMWH arm. ${ }^{58}$ Large treatment trials in pediatrics and AYA patients with VTE and cancer are not available. Nonetheless, gastrointestinal issues and drug-drug interactions, as well as treatment interruptions for invasive procedures, present challenges to the use of oral anticoagulation, and it is not unreasonable to consider an LMWH-based regimen in patients who may be subject to these complications.

New oral anticoagulants, including rivaroxaban, apixaban, and dabigatran, are now widely used as VTE therapy in 
noncancer adult patients. Prospective randomized trials comparing these agents with LMWH in adult cancer patient are in progress, but data to this point are restricted to retrospective observational studies, which suggest that rivaroxaban may be of comparable safety and efficacy to LMWH, at least in adults. ${ }^{59,60}$ However, it should be noted that the rate of recurrent VTE in these studies was quite low, suggesting that the patients treated with rivaroxaban in these studies may not be reflective of the average cancer patient on chemotherapy. This is also highlighted in a meta-analysis of the subgroups of patients included in the large randomized trials on the new direct oral anticoagulants (DOACs) who were classified as having cancer (definitions of cancer varied between trials). This analysis showed that while DOACs appeared similar in efficacy and safety when compared to warfarin, there were substantially fewer recurrent thrombotic events in the warfarin arms of the DOAC trials compared to the warfarin arms of the LMWH trials. This underscores that these data cannot be applied to "real world" cancer patients yet. ${ }^{61}$

DOACs are subject to many potential problems in the cancer patient. These include altered gastrointestinal function from either the cancer itself, the resulting cachexia, nausea/vomiting, or gut lining damage from chemotherapy. This may result in poor and unpredictable absorption of oral medications. There are also a host of drug interactions from chemotherapy and supportive care such as antibiotics, antiemetics, and analgesia. Therefore, until prospective randomized trials are available, DOACs cannot currently be recommended routinely for cancer patients and, in particular, should be used with extreme caution in patients receiving antineoplastic and supportive care agents, which may interact with them. This is more important in the younger AYA population as data on DOACs even in the noncancer pediatric population are lacking.

\section{How should patients with renal failure be managed?}

Patients with renal failure pose a challenge. Baseline risk of bleeding is higher in patients with renal impairment irrespective of anticoagulant use. ${ }^{62-64}$ While LMWH is often the treatment of choice in cancer-associated thrombosis, all LMWHs rely to some extent on renal clearance. The use of LMWH in those with significant renal impairment may be associated with drug accumulation and an increased risk of bleeding. LMWHs that are larger and more heavily charged (tinzaparin, dalteparin) compared to those that are smaller (enoxaparin) are predicted to be less dependent on renal clearance. ${ }^{65}$ There are no large trials specifically addressing this issue in any population of patients, but there are some studies that have attempted to measure potential accumulation using anti-Xa level monitoring. It appears that prophylactic doses of dalteparin and tinzaparin do not accumulate even in patients with severe renal failure. ${ }^{65,66}$ Subgroup analysis of the IRIS study showed that therapeutic tinzaparin used to treat DVT in their elderly population did not accumulate despite moderate renal failure. ${ }^{67}$ A post hoc analysis of the CLOT trial revealed that $24 \%$ of patients had renal impairment at baseline, with $2.2 \%$ having severe renal impairment (creatinine clearance $<30 \mathrm{~mL} / \mathrm{min}$ ). Superiority of dalteparin was maintained in the renal-impaired subgroup, with no statistically significant increase in major bleeding $(9.5 \%$ with dalteparin versus $6.9 \%$ with warfarin, $P=0.65){ }^{68}$

However, drug accumulation and increased rates of bleeding with enoxaparin do appear to be higher in patients with renal failure, and this LMWH should be avoided in the setting of severe renal failure. ${ }^{69}$ Given the paucity of data on LMWH in cancer patients with renal failure, caution and appropriate counseling and clinical follow-up should be employed when using LMWH in those with moderate renal impairment (creatinine clearance $30-60 \mathrm{~mL} / \mathrm{min}$ ). Unless under the guidance of an expert nephrologist/thrombosis specialist, LMWH should be avoided in patients with a creatinine clearance of $<30 \mathrm{~mL} / \mathrm{min}$, and unfractionated heparin bridged to warfarin therapy may be used as a substitute. If LMWH is used in this setting, checking anti-Xa trough levels (target $<0.4$ units $/ \mathrm{mL}$ ) to monitor for accumulation has been suggested for adult patients. $^{70}$

\section{How should VTE be treated in patients at high risk of bleeding?}

Anticoagulation use must be balanced against the risk of bleeding. There is a remarkable paucity of data surrounding bleeding and predictors of bleeding in VTE patients on anticoagulation particularly in the cancer populations. In noncancer adult trials on VTE treatment, major bleeding is estimated to be around $1 \%-2 \%$ per year, though this increases in population-based studies and "real world" data depend on factors such as age, comorbidities, and concurrent medications such as nonsteroidal anti-inflammatories and antiplatelet agents. ${ }^{48}$ In adult cancer-associated thrombosis trials, major bleeding rates in active cancer patients were around $5 \% .{ }^{53}$ This highlights that while cancer patients are at higher thrombotic risk, they are also at higher bleeding risk. This is likely secondary to a higher concurrent rate of gastrointestinal lesions, vascular tumors, thrombocytopenia from the cancer or its treatment, and other major organ dysfunction 
such as renal failure. As the rate of recurrent VTE is 50\% in the first 3 months ( $40 \%$ in the first month), in the absence of major bleeding, benefits of anticoagulation for acute VTE still outweigh these risks. ${ }^{8}$ Caution, appropriate counseling, and monitoring of cancer patients should be employed given the higher risk of complications. While there are no AYA and pediatric specific trials to counterpoint the adult data, principles of balancing thrombotic and hemorrhagic risk when selecting the appropriate treatment for VTE cancer patients are important considerations.

For patients with moderate-to-severe thrombocytopenia, the elevated bleeding risk needs to be factored into the decision-making. Although there are no prospective studies to address the optimal approach, some experts recommend lowering the dose of LMWH in thrombocytopenic patients. A retrospective analysis of such an approach was recently reported from Memorial Sloan Kettering Cancer Center, ${ }^{71}$ in which patients with platelet counts between $25 \times 10^{9}$ and $50 \times 10^{9} / \mathrm{L}$ had a $50 \%$ dose reduction in the dose of enoxaparin, while the dose was temporarily stopped if the platelet count dropped below 25. Of 144 thrombocytopenic events in 101 patients, there was only one major bleeding event, and no recurrent VTE.

An alternative approach, particularly in the setting of acute VTE and severe thrombocytopenia, would be to continue LMWH with aggressive prophylactic platelet transfusion support to maintain the platelet count above 25-30. A similar strategy is endorsed by a recent Canadian expert opinion consensus recommendation on "clinical challenges" in adult patients with cancer-associated thrombosis. ${ }^{70}$ In addition to potential LMWH dose reduction depending on platelet count and the use of platelet transfusions as needed, it is importantly highlighted that the timing from the thrombotic event ( $<1$ month versus $\geq 1$ month) should also factor into the decision as the risk of recurrent thrombosis is substantially smaller when the acute phase of clot evolution has passed. The decision to lower the dose of myelosuppressive (ie, platelet lowering) chemotherapy agents should depend on whether the treatment is being administered with curative intent. In such cases, most experts would recommend maintaining dose intensity of these drugs and using platelet transfusion support, while in the palliative setting a chemotherapy dose reduction would be reasonable to minimize the increased bleeding risk.

With respect to the use of inferior vena cava filters for patients with lower extremity DVT, a recent retrospective study in adult cancer patients with a variety of malignancies indicates that these do not necessarily protect against $\mathrm{PE}$ and are associated with a higher rate of recurrent DVT and increased risk of complications. ${ }^{72}$ Although there are no comparable data in AYA patients, these findings suggest caution in their use in cancer patients in general; their use should be restricted to situations where an acute lower extremity DVT is present and anticoagulation is absolutely contraindicated (eg, severe active bleeding), or if the platelet count cannot be maintained above $20 \times 10^{9} / \mathrm{L}$ even with transfusion support (eg, platelet alloimmunization).

\section{What is the optimal management of central venous catheter-associated venous clots?}

Catheter-associated upper extremity thrombosis is a common complication in cancer patients and can be found in $14 \%-18 \%$ on screening imaging studies, with $\sim 5 \%$ being symptomatic. ${ }^{73}$ Unfortunately, because upper extremity DVT only comprises approximately $5 \%-10 \%$ of all VTE, ${ }^{51}$ there are less data available to guide management. The initial assessment typically depends on the location of the clot Generally speaking, deep veins in the arm are classified as including the axillary vein and more proximal, though the brachial vein has also been included as a deep vein in some studies. Smaller veins (cephalic, basilic, and other more distal/superficial arm veins) are less likely to extend or embolize and treatment of thrombosis found in these veins is controversial with a tendency to not anticoagulate. ${ }^{70}$ Upper extremity DVT (axillary vein and more proximal) has general epidemiologic data in adult patients, which include a $5 \%$ association with $\mathrm{PE}, 8 \%$ recurrence rate in 5 years, and $20 \%$ postthrombotic syndrome development. ${ }^{48}$ The presence of a catheter and/or cancer increases the risk of upper extremity DVT and can be ongoing risk factors after a thrombotic event occurs.

It thus stands to reason that in the highly thrombogenic cancer patient, anticoagulation should also be recommended if a catheter-associated DVT develops. Duration of anticoagulation is controversial but is for a minimum duration of 3 months, largely extrapolated from lower extremity DVT and $\mathrm{PE}$ data. ${ }^{48}$ If the central venous catheter remains in place, such as for ongoing chemotherapy, expert opinion would recommend continuing anticoagulation as thrombotic risk factors persist. If the line is removed at or before 3 months, anticoagulant cessation can be considered after the 3-month mark. This is consistent with existing general adult thrombosis guidelines and expert panel consensus opinions. Finally, there is reasonable evidence to support leaving the catheter in place as long as it is still required and functioning rather than removing/replacing it after DVT. ${ }^{74}$ 


\section{How should asparaginase-associated VTE be managed?}

There is published expert opinion-level evidence for the management of AYAs who are being treated with asparaginase for ALL to prevent and manage VTE. ${ }^{75}$ For AYAs, the incidence of thrombosis is known to be increased compared to that of children receiving similar therapy, ${ }^{23}$ with the most likely time for thrombosis occurring during either induction ${ }^{76}$ or in the postremission intensification phase, ${ }^{32,77}$ depending on the chemotherapy protocol used. Limited evidence is available from pediatric studies to support the prophylactic use of antithrombin III concentrate supplementation during induction ${ }^{78}$ or anticoagulation with LMWH. ${ }^{79}$ In the latter retrospective study, the use of enoxaparin $1 \mathrm{mg} / \mathrm{kg} / \mathrm{d}$ was associated with a significantly lower risk of VTE. In contrast, one retrospective analysis in adults did not demonstrate a reduced incidence of VTE using prophylactic fixed low-dose enoxaparin during postremission asparaginase-containing therapy; however, patients with dose-adjusted enoxaparin at $1 \mathrm{mg} / \mathrm{kg} / \mathrm{d}$ trended toward a lower VTE rate. ${ }^{80}$ The data, therefore, suggest that adjusted dose LMWH may be preferred for such higher-risk patients. However, this is based on retrospective data, and prospective studies are needed to verify this.

If clinically significant VTE or central nervous system thrombosis occurs, the patient should be anticoagulated, with or without antithrombin III supplementation. The asparaginase should be temporarily stopped, but may be resumed once the patient has been stabilized on anticoagulation. Permanently discontinuing asparaginase in patients with uncomplicated VTE is not advised due to the potential increased risk of relapsed leukemia; several studies have shown a lower event-free survival and higher cumulative incidence of relapse in patients discontinuing asparaginase early due to toxicity. 32,77

\section{What is the optimal duration of anticoagulation?}

The minimum duration of anticoagulation for a proximal DVT and/or PE has been established in the adult population as 3 months as recurrence rates are very high when duration is shorter (ie, the acute thrombosis has not yet healed) and does not change thereafter no matter what the duration of anticoagulation is beyond 3 months (ie, the acute clot has healed and thrombotic events now reflect new recurrences which in turn reflect the patient's thrombotic risk). ${ }^{81}$ While these data were generated from the general population and not cancer-specific, it can be logically extrapolated that as cancer confers an even higher thrombotic risk, the minimum duration of therapy would not be shorter than 3 months. Duration beyond 3 months is currently left up to the discretion of the treating physician. Adult cancer-associated thrombosis trials have generally used an arbitrary 6-month time frame in their protocols, ${ }^{53,57}$ and this has resulted in a typical treatment period extended to at least 6 months, but with a lack of data on safety and efficacy of anticoagulation beyond this time point.

Duration of anticoagulation is typically recommended to be "defined" (ie, short term such as only 3 months) in those with transient provoking risk factors, who are predicted to have a low risk of recurrence, or who are predicted to have a high risk of bleeding. Duration is conversely recommended to be "indefinite" (ie, long term with no specified stop date) in those with unprovoked VTE, who have permanent strong risk factors for VTE, or who have low bleeding risk. ${ }^{82}$ This speaks to the importance of balancing thrombotic risk while off anticoagulation with major bleeding risk while continuing on anticoagulation. As such, it is reasonable to apply this to cancer patients and derive expert opinion-based recommendations. At the 3-month point, an assessment of thrombotic and bleeding risk should be done. Assuming there are no contraindications to ongoing anticoagulation, if the cancer remains active and/or active treatment such as chemotherapy continues, extending anticoagulation to cover the ongoing risk is recommended. If the cancer is considered cured and no further therapy is required, it may be reasonable to discuss anticoagulant cessation. If cancer is palliative in nature, there may be benefit to continuing anticoagulation even if chemotherapy is no longer given, as long as preventing recurrent VTE still positively impacts on quality of life. In patients with cancers that are incurable but can enter long periods of remission/disease-free states, anticoagulant management often relies on patient and doctor preference. If bleeding risk is low, it is not unreasonable to continue anticoagulation during remission periods as sometimes the first sign of cancer recurrence can be thrombotic events, which add to morbidity and mortality. Oral anticoagulant options may be considered in the surveillance phase.

\section{Conclusion}

Thromboembolism is a serious complication for AYAs with cancer. The paucity of literature regarding the true incidence and risk factors of VTE within AYA oncology, as well as management strategies for this population to both prevent and treat thrombosis, create a challenge for the clinician. While it is well recognized that AYAs with cancer do not fit perfectly into either the pediatric or adult physiology and pharmacology due to issues of development, disease biology, and patient-specific 
factors, the current practice is to manage AYAs with cancer and VTE as either a child or an adult depending on their age and the center where they are being treated. At present, balancing the risks of thrombosis and bleeding in establishing a treatment plan for the AYA oncology patient must remain a guiding principle. By consensus between adult and pediatric literature, the use of LMWH for the management of acute VTE should be considered the preferred anticoagulant. This may change in the coming years as results from prospective studies using new oral anticoagulants in cancer patients become available.

Specific risk factors predisposing AYAs with cancer to thrombosis require further investigation, considering the complexities of the coagulation activation due to the cancer, treatment, and patient-specific factors. Prospective collaborative studies with specific attention to the AYA population are needed to improve the understanding of AYA thrombotic risk factors. The further development and validation of prediction models for the development of thrombosis in this population as well as validation of diagnostic modalities for AYA thrombosis will allow for better development of prophylactic as well as therapeutic strategies for the management of VTE in AYA oncology.

\section{Disclosure}

CW received honoraria from Pfizer and Leo Pharma for advisory board meetings and speaking engagements. The authors report no other conflicts of interest in this work.

\section{References}

1. Bouillard S. [De l'Obliteration des veins et de son influence sur la formation des hydropisies partielles: consideration sur las hydropisies passive et general]. Arch Gen Med. 1823;1:188-204. French.

2. Trousseau A, Bazire PV, Cormack JR. Lectures on Clinical Medicine. London, England: R Hardwicke; 1967.

3. Timp J, Braekkan S, Versteeg, H., Cannegieter S. Epidemiology of cancer-associated venous thrombosis. Blood. 2013;122(10):1712-1723.

4. Khorana A, Francis C, Culakova E, et al. Thromboembolism is a leading cause of death in cancer patients receiving outpatient chemotherapy. $J$ Thromb Haemost. 2007;5(3):632-634.

5. National Comprehensive Cancer Network. NCCN clinical practice guidelines in oncology: venous thromboembolic disease 2015. Available from: http://www.nccn.org/professionals/physician_gls/pdf/vte. pdf. Accessed December 14, 2015.

6. Beckman M, Hooper W, Critchley S, Ortel T. Venous thrombosis. Am J Prev Med. 2010;38(4):S495-S501.

7. Engbers M, van Hylckama Vlieg S, Rosendaal F. Venous thrombosis in the elderly: incidence, risk factors and risk groups. J Thromb Haemost. 2010;8(10):2105-2112.

8. Kearon C. Natural history of venous thromboembolism. Circulation. 2003;107(23 Suppl 1):I22-130.

9. Silverstein M, Hejt J, Mohr D, Petterson T, O’Fallon W, Melton L. Trends in the incidence of deep vein thrombosis and pulmonary embolism: a 25 year population study. Arch Intern Med. 1998;158(6):585-593.

10. White R. The epidemiology of venous thromboembolism. Circulation. 2003;107(23 Suppl 1):I4-I8.

11. Pioversen D, Attard C, Monagle P, Ignjatovic V. Epidemiology of venous thrombosis in children with cancer. Thromb Hemost. 2014;111:1015-1021.
12. Raffin, L, Huang Y, Witmer C, et al. Dramatic increase in venous thromboembolism in children's hospitals in the United States from 2001 to 2007. Pediatrics. 2009;124:1001-1008.

13. Andrew M, David M, Adams M, et al. Venous thromboembolic complications (VTE) in children: first analysis of Canadian registry of VTE. Blood. 1994;83:1251-1257.

14. Setty, B, O'Brien S, Kerlin B. Pediatric venous thromboembolism in the United States: a tertiary care complication of chronic diseases. Pediatr Blood Cancer. 2011;59(2):258-264.

15. Vu L, Nobuhara K, Lee H, et al. Determination of risk factors for deep venous thrombosis in hospitalized children. J Pediatr Surg. 2008;43:1095-1099.

16. Stein P, Beemath A, Meyers F, et al. Incidence of venous thromboembolism in patients hospitalized with cancer. Am J Med. 2006;119(1): 60-68.

17. Ageno W, Squizzato A, Garcia D, Imberti D. Epidemiology and risk factors of venous thromboembolism. Semin Thromb Hemost. 2006;32(7):651-658.

18. Monreal M, Falga M, Valdes C, et al. Fatal pulmonary embolism and fatal bleeding in cancer patients with venous thromboembolism: findings from the RIETE registry. J Thromb Haemost. 2006;4(9):1950-1956.

19. O'Brien S, Klima J, Termuhlen A, Kelleher K. Venous thromboembolism and adolescent and young adult oncology inpatients in US children's hospitals, 2001-2008. J Pediatr. 2011;159(1):133-137.

20. Panessha S, McManus A, Arya R, et al. Frequency, demographics and risk (according to tumor or site) of cancer-association thrombosis among patients seen at outpatient DVT clinics. Thromb Haemost. 2010;103(2):338-343.

21. Kayser-Gatchalian M, Kayser, K. Thrombosis and intracranial tumors J Neurol. 1975;209(3):217-224.

22. Audino A, Yeager N, Asti L, Miao Y, O'Brien S. Length of stay and treatment-related complications are similar in pediatric and AYA patients with bone sarcoma in the United States children's hospitals. Pediatr Blood Cancer. 2013;60(3):415-419.

23. Grace R, Dahling S, Neuberg D, et al. The frequency and management of asparaginase-related thrombosis in paediatric and adult patients with acute lymphoblastic leukaemia treated on Dana-Farber Cancer Institute consortium protocols. Br J Haematol. 2011;152(4):452-459.

24. Paz-Priel I, Long L, Helman L, Mackall C, Wayne A. Thromboembolic events in children and young adults with pediatric sarcoma. J Clin Oncol. 2007;25(12):1519-1924.

25. Chew H, Wun T, Harvey D, et al. Incidence of venous thromboembolism and its effect on survival among patients with common cancers. Arch Intern Med. 2006;166(4):458-464.

26. Falanga A. Pathophysiology of the thrombophilic state in the cancer patient. Semin Thromb Hemost. 1999;25(2):173-182.

27. Kuderer N, Ortel T, Francis C. Impact of venous thromboembolism and anticoagulation on cancer and cancer survival. J Clin Oncol. 2009;27(29):4902-4911.

28. Rickels F. Mechanisms of cancer-induced thrombosis. J Pathophysiol Haemost Thromb. 2006;35(1-2):103.

29. Saber W, Moua T, Williams E, et al. Risk factors for catheter-related thrombosis (CRT) in cancer patients: a patient-level data (IPD) metaanalysis of clinical trials and prospective studies. J Thromb Haemost. 2011;9:312-319.

30. Lichman H, Wada J, Patch M, McGehee W. Depression of functional and antigenic plasma antithrombin III due to therapy with L-asparaginase. Cancer. 1982;50(3):451-456.

31. Boissel N, Sender L. Best practices in adolescent and young adult patients with acute lymphoblastic leukemia: a focus on asparaginase. J Adolesc Young Adult Oncol. 2015;4(3):118-128.

32. Storring JM, Minden MD, Kao S, et al. Treatment of adults with BCRABL negative acute lymphoblastic leukemia (ALL) with a modified pediatric regimen. Br J Haematol. 2009;146(1):76-85.

33. Ploude P, Jeha S, Hijiya N, et al. Safety profile of asparaginase Erwinia chrysanthemi in a large compassionate use trial. Pediatr Blood Cancer. 2014;61(7):1232-1238. 
34. Khorana A, Kuderer N, Culakova, E, et al. Development and validation of a predictive model for chemotherapy associated thrombosis. Blood. 2008;111(10):4902-4907.

35. Bell A, Hockenberry M, Landier W, Ewing N. Venous thromboembolism risk assessment and prophylaxis use in pediatric, adolescent and young adult hematology oncology patients. J Pediatr Hematol Oncol. 2015;37(6):455-458.

36. Prentiss A. Pediatric venous thromboembolism (VTE) risk assessment tool. 2010. All publications. Paper 922. Available at: http://scholarlycommons.baptisthealth.net/se-all-publications/922. Accessed June 23, 2016.

37. Athale U. Thrombosis in pediatric cancer: identifying the risk factors to improve care. Expert Rev Hematol. 2013;6(5):599-609.

38. Chan A, Deveber G., Monagle P, et al. Venous thrombosis in children. J Thromb Haemost. 2003;1(7):1443-1455.

39. Chalmers E, Ganesen V, Liesner R, et al. Guidelines on the investigation, management and prevention of venous thrombosis in children. $\mathrm{Br}$ J Haematol. 2011;154(2):196-207.

40. Male C, Chart P, Ginsberg J, et al. Comparison of venography and ultrasound for the diagnosis of asymptomatic deep vein thrombosis in the upper body in children: the results of the PARKAA study. Prophylactic antithrombin replacement in kids with ALL treated with asparaginase. Thromb Hemost. 2002;87(4):593-598.

41. Wells P, Hirsh J, Anderson D, et al. Accuracy of clinical assessment of deep-venous thrombosis. Lancet. 1995;345(8961):1326-1330.

42. Wells P, Ginsberg J, Anderson D, et al. Use of clinical model for safe management of patients with suspected pulmonary embolism. Ann Intern Med. 1998;129(12):997-1005.

43. Kearon C, Ginsberg J, Douketis J, et al. An evaluation of D-dimer in the diagnosis of pulmonary embolism: a randomized trial. Ann Intern Med. 2006;144(11):812-821.

44. Kearon C, Julian J, Newman T, et al. Noninvasive diagnosis of deep vein thrombosis. Ann Intern Med. 1998;128(8):663-677.

45. Kearon C. Diagnosis of pulmonary embolism. Can Med Assoc J. 2003;168(2):183-194.

46. PIOPED investigators. Value of the ventilation/perfusion scan in acute pulmonary embolism; results of the prospective investigation of pulmonary embolism diagnosis. J Am Med Assoc. 1990;263(20):2753-2759.

47. Khorana A, O'Connell C, Agnelli G, et al. Incidental venous thromboembolism in oncology patients. J Thromb Haemost. 2012;10(12):2602-2604.

48. Kearon C, Comerota A, Prandoni P, et al. Antithrombotic therapy for VTE disease: antithrombotic therapy and prevention of thrombosis. 9th ed: American college of chest physicians evidence-based clinical practice guidelines. Chest. 2012;14(2 Suppl):e419S-e494S

49. Streiff M, Carolan H, Hobson D, et al. Lessons from the Johns Hopkins multi-disciplinary venous thromboembolism (VTE) prevention collaboration. Br J Med. 2012;344:e3935.

50. Sharma M, Carpenter S. Thromboprophylaxis in a pediatric hospital. Curr Probl Pediatr Adolesc Health Care. 2013;43(7):178-183.

51. Easaw JC, Shea-Budgell MA, Wu CM, et al. Canadian consensus recommendations on the management of venous thromboembolism in patients with cancer. Part 1: prophylaxis. Curr Oncol. 2015;22(2):133-143.

52. Monagle $\mathrm{P}$, Chan A, Goldenberg N, el al. Antithrombotic therapy in neonates and children. Chest. 2012;141(2 Suppl):e737S.

53. Lee A, Levine M, Baker R, et al. Low-molecular-weight heparin versus Coumadin for the prevention of recurrent venous thromboembolism in patients with cancer. N Engl J Med. 2003;349(2):146-153.

54. Hull R, Pineo G, Brant R, et al. Long-term low-molecular weight heparin versus usual care in proximal-vein thrombosis patients with cancer. $\mathrm{Am}$ J Med. 2006;119(2):1062-1072.

55. Ribera J, Oriol A. Acute lymphoblastic leukemia in adolescents and young adults. Hematol Oncol Clin N Amer. 2009;23(5):1033-1042.

56. Verrill M, Judson I, Wiltshaw E, et al. The use of paediatric chemotherapy protocols at full dose is both a rational and feasible treatment strategy in adults with Ewing's family tumors. Ann Oncol. 1997;8(11):1099-1105.

57. Lee AY, Kamphuisen P, Meyer G, et al. Tinzaparin vs warfarin for treatment of acute venous thromboembolism in patients with active cancer. J Am Med Assoc. 2015;314(7):677-686.
58. Massicotte P, Julian J, Gent M, et al. An open label randomized control trial of low molecular weight heparin and Coumadin for the treatment of venous thromboembolic events in children: the REVIVE trial. Thromb Res. 2003;109(2-3):85-92.

59. Chaudbury A, Balakrishnan A, Thai C, et al. Evaluation of rivaroxaban and dalteparin in cancer associated thrombosis. Blood. 2015; 126(23):432a.

60. Win KZ, Wilson N, Stenehjem DD, et al. Effectiveness and safety of rivaroxaban in treatment of venous thromboembolism in cancer patients, Blood. 2015;126(23):2319a.

61. Carrier M, Cameron C, Delluc A, Castellucci L, Khorana AA, Lee AYY. Efficacy and safety of anticoagulant therapy for the treatment of acute cancer-associated thrombosis: a systematic review and meta-analysis. Thromb Res. 2014;134(6):1214-1219.

62. Falga C, Capdevila J, Soler S, et al. Clinical outcome of patients with venous thromboembolism and renal insufficiency: findings from the RIETE registry. Thromb Haemost. 2007;98(4):771-776.

63. Lim W, Dentali F, Eikelboom J, et al. Meta-analysis: low molecularweight heparin and bleeding in patients with severe renal insufficiency. Ann Intern Med. 2006;144(9):673-684.

64. Capodanno D, Angiolillo D. Antithrombotic therapy in patients with chronic kidney disease. Circulation. 2012;125(21):2649-2661.

65. Trujillo-Santos J, Schellong S, Falga C, et al. Low-molecular-weight or unfractionated heparin in venous thromboembolism: the influence of renal function. Am J Med. 2013;126(5):425-434.e1

66. Lim W. Low-molecular-weight heparin in patients with chronic renal insufficiency. Intern Emerg Med. 2008;3(4):319-323.

67. Leizorovicz A, Siguret V, Mottier D. Safety profile of tinzaparin versus subcutaneous unfractionated heparin in elderly patients with impaired renal function treated for acute deep vein thrombosis: the Innohep in Renal Insufficiency Study (IRIS). Thromb Res. 2011;128(1): 27-34.

68. Woodruff S, Feuge're G, Abreu P, Heissler J, Jen F. A post-hoc analysis of dalteparin versus oral anticoagulation (VKA) therapy for the prevention of recurrent venous thromboembolism (rVTE) in patients with cancer and renal impairment. J Thromb Thrombolysis. 2015;39(3):418.

69. Hoffman P, Keller F. Increased major bleeding risk in patients with kidney dysfunction receiving enoxaparin: a meta-analysis. Eur J Clin Pharmacol. 2012;68(5):757-765.

70. Carrier M, Lazo-Langner A, Shivakumar S, et al. Clinical challenges in patients with cancer-associated thrombosis: Canadian expert consensus recommendations. Curr Oncol. 2015;22(1):49-59.

71. Miao Y, Soff GA, Parameswaran R, et al. Enoxaparin dose reduction for thrombocytopenia in patients with cancer: a quality assessment study. Blood. 2015;126(23):429a.

72. Coombs CC, Kuk D, Devlin S, et al. Outcomes of inferior vena cava filter placement in a large population of cancer patients diagnosed with pulmonary embolism: risk for recurrent venous thromboembolism, survival and filter-related complications. Blood. 2015;126(23):1112a.

73. Lee A, Kamphuisen P. Epidemiology and prevention of catheterrelated thrombosis in patients with cancer. J Thromb Haemost. 2012;10(8):1491-1499.

74. Kovacs MJ, Kahn SR, Rodger M, et al. A pilot study of central venous catheter survival in cancer patients using low-molecular-weight heparin (dalteparin) and warfarin without catheter removal for treatment of upper extremity deep vein thrombosis (The Catheter Study). J Thromb Haemost. 2007;5(8):1650-1653.

75. Stock W, Douer D, DeAngelo D, et al. Prevention and management of asparaginase/pegasparaginase-associated toxicities in adults and older adolescents: recommendations of an expert panel. Leuk Lymphoma. 2011;52(12):2237-2253.

76. Caruso V, Iacoiello L, Dicastelnuovo A, et al. Venous thrombotic complications in adults undergoing induction treatment for acute lymphoblastic leukemia: results from a meta analysis. J Thromb Haemost. 2007;5(3): 621-623.

77. Silverman LB, Gelber RD, Kimball Dalton V, et al. Improved outcome for children with acute lymphoblastic leukemia: results of Dana Farber Consortium Protocol 91-01. Blood. 2001;97(5):1211-1218. 
78. Mitchell LG, Andrew M, Hanna K, et al. A prospective cohort study determining the presence of thrombotic events in children with acute lymphoblastic leukemia and a central venous line who are treated with L-asparaginase: results of the PARKAA study. Cancer. 2003;97(2): 508-516.

79. Mitchell L, Lambers M, Flege S, et al. Validation of a predictive model for identifying an increased risk for thromboembolism in children with acute lymphoblastic leukemia: results of a multicentre cohort study. Blood. 2010;115(40):4999-5004.
80. Sibai H, Seki JT, Atenafu EG, et al. Anticoagulation prophylaxis for patients receiving asparaginase-based intensification therapy for acute lymphoblastic leukemia. Curr Oncol. In press.

81. Boutitie F, Pinede L, Schulman S, et al. Influence of preceding length of anticoagulant treatment and initial presentation of venous thromboembolism on risk of recurrence after stopping treatment: analysis of individual participants' data from seven trials. Br Med J. 2011;342:d3036.

82. Kearon C, Akl E. Duration of anticoagulation for deep vein thrombosis and pulmonary embolism. Blood. 2014;123(12):1794-1801.
Clinical Oncology in Adolescents and Young Adults is an international, peer-reviewed, open access journal publishing origina research, reports, editorials, reviews and commentaries on all aspects of epidemiology, diagnosis and treatment of cancers in adolescents and young adults. Visit http://www.dovepress.com/testimonials.php to read real quotes from published authors. 